\title{
First nationwide web-based surveillance system for influenza-like illness in pregnant women: participation and representativeness of the French G-GrippeNet cohort
}

Paul Loubet ${ }^{1,2}$, Caroline Guerrisi ${ }^{1}$, Clément Turbelin ${ }^{1}$, Béatrice Blondel ${ }^{3}$, Odile Launay ${ }^{2,3,4,5}$, Marc Bardou ${ }^{6}$, Thierry Blanchon ${ }^{1}$, Isabelle Bonmarin ${ }^{7}$, François Goffinet ${ }^{3,8}$, Pierre-Yves Ancel ${ }^{3}$, Vittoria Colizza',

Thomas Hanslik ${ }^{1}$ and Solen Kernéis $2,3,4^{*}$

\begin{abstract}
Background: Pregnancy is a risk factor for severe influenza resulting in increased risks of hospitalisation and death in mothers and their new-borns. Our objective was to assess the representativeness and participation of French women to a new web-based collaborative tool for data collection and monitoring of Influenza Like Illness (ILI) during pregnancy.

Methods: During the 2014/2015 influenza season, pregnant women living in metropolitan France were enrolled through a web platform (https://www.grippenet.fr/). Then throughout the season, participants were asked to report, on a weekly basis, if they had experienced symptoms of ILI. Representativeness was assessed by comparing the characteristics of participants to those of the French National Perinatal Survey. For each participant, the participation rate was the number of weekly questionnaires completed, divided by the length of follow-up (in weeks). Predictors of active participation (participation rate $>15 \%$ ) were assessed by multivariate logistic regression.

Results: A total of 153 women were enrolled. Participants were older (mean age 34 years vs. 29 years) and more highly educated (high school level $89 \%$ versus $52 \%$ ) than the general population of pregnant women in France, but the sample did not differ on pregnancy-related characteristics (parity, history of hospitalisation during a previous pregnancy). The median rate of participation was high (78 \%, interquartile range: 34-96). Higher educational level and participation to a previous GrippeNet.fr season were associated with active participation.
\end{abstract}

Conclusion: Despite small sample size and lack of representativeness, the retention rate was high, suggesting that pregnant women are prone to adhere to a longitudinal follow-up of their health status via the Internet.

Keywords: Pregnancy, Influenza, Incidence, Surveillance, Web-based study

\footnotetext{
* Correspondence: solen.kerneis@aphp.fr

${ }^{2}$ Department of Infectious diseases, CIC Cochin-Pasteur, Cochin Broca

Hôtel-Dieu hospital, Assistance Publique-Hôpitaux de Paris (AP-HP), Paris,

France

${ }^{3}$ INSERM U 1153, Obstetrical, Perinatal and Pediatric Epidemiology Research

Team (EPOPé), Center for Epidemiology and Statistics Sorbonne Paris Cité,

DHU Risks in pregnancy, Paris Descartes University, Paris, France

Full list of author information is available at the end of the article
} 


\section{Background}

Some infections are more severe in pregnant women and most may also harm the fetus or the new-born. This is the case for influenza that results in higher risks for hospitalisation and death in pregnant women even in the absence of underlying comorbidities [1-3]. Influenza also increases the risks of miscarriage, low birth weight, and prematurity [4-6].

Seasonal influenza surveillance systems provide essential information to describe the diffusion of the epidemics and monitor the incidence through the season [7]. In France, the surveillance is traditionally based on health care systems, excluding people who do not consult for influenzalike illness (ILI) [8, 9]. New technologies and widening of the Internet coverage have opened the way to real-time collaborative data collection and led to the development of innovative approaches for disease surveillance [10, 11]. In 2011, a nationwide surveillance system for ILI, GrippeNet.fr was introduced in France. It is based on the online participation of volunteers from the general population and allows us to gather data on incidence of ILI in the community, and on vaccination coverage, vaccine effectiveness and health-care usage [12, 13]. The participation steadily increased from the first season to reach 6632 participants in 2014/2015.

In pregnant women and women who plan a pregnancy, several web-based studies have already been undertaken, mainly focusing on pre-conception risk factors for adverse pregnancy outcomes [14-18]. Regarding influenza, only one annual transversal web-based survey is conducted each year since $2010 / 2011$ in the USA to assess vaccination coverage among pregnant women $[19,20]$. No specific realtime surveillance of ILI in pregnant women is conducted, to our knowledge, and the incidence of the disease is not well documented in this population.

The G-GrippeNet (Grossesse-GrippeNet in French, Pregnancy-GrippeNet) project aimed to describe the epidemiology of seasonal influenza during the influenza season among pregnant women in France. Objectives of the present study were to report data on representativeness of the cohort and factors associated with participation for the opening season 2014/2015.

\section{Methods}

\section{Study design}

The G-GrippeNet project, dedicated to pregnant women, hereafter referred to as GGNET, was integrated into the national GrippeNet.fr web-based annual campaign conducted between November 19th 2014 and April 14th 2015. As described previously [12], GrippeNet.fr (https://www.grippenet.fr/) is an online surveillance project integrated to the European project Influenzanet $[21,22]$. Participants are volunteers passively recruited (after communication actions on television, radio, newspapers and others (Influenzanet), poster, and leaflets) in the general population. They register, through their e-mail address, directly on the GrippeNet.fr website. After inclusion, participants are invited to fill in a first questionnaire dealing with general background information. Then, they are invited to self-report on a weekly basis on their health status, reporting easily identifiable symptoms of influenza or stating the absence of symptoms.

\section{Inclusion criteria}

Participants to GGNET had to: 1) be pregnant (regardless of the trimester) 2) be resident in mainland France; 3) read French language; 4) have active email address and Internet access.

\section{Data collection}

Women enrolled were followed throughout the influenza season and asked weekly whether or not they experienced any influenza-like illness. ILI was defined as the combination of the three following criteria: (i) the sudden onset of symptoms, (ii) at least one of the following signs: fever or chills or headache or myalgia or asthenia and (iii) at least one of the following respiratory symptoms: cough, sore throat, shortness of breath (dyspnea) [9].

The intake questionnaire covered age, household size and composition, location of home and workplace, education level, occupation, vaccination status for the previous and present influenza season, the presence of a chronic disease that may lead to more severe influenza case (i.e. chronic respiratory or cardiac diseases, diabetes, and immunosuppression) [12]. Specific questions designed for pregnant women, added to the questionnaire, included pregnancy related factors such as gravidity, parity, estimated date of delivery, history of hospitalisation during a previous pregnancy. Participants were reminded weekly, via an email newsletter, to report through a brief weekly questionnaire (WQ) whether or not they experienced any ILI symptoms since the last seven days. All questionnaires were self-administered. In addition, every month, participants received newsletters on prevention and treatment of infectious diseases during pregnancy, which were used to maintain a communication channel with each participant as a motivation purpose during the season.

\section{Recruitment of participants}

We promoted the project through articles published on web sites dedicated to pregnant women and young mothers' health. Two national clinical research networks (iREIVAC and GO-CIC), including 30 maternity centres, were also solicited by the investigators. Among them, 10 agreed to participate and promoted the study by displaying advertising posters in their waiting rooms. These 10 maternity centres accounted for $4 \%$ of the total number of delivery per year in the country. No incentives 
were offered for participation in the study. Consent to participation to the study was obtained through an online form.

\section{Statistical analysis}

Coverage was calculated by dividing the number of participants to GGNET by the average number of pregnant women in France over the study period. The latter was based on national statistics [23], and calculated by summing the number of ingoing pregnancies at the beginning of the study to the number of new pregnancies occurring during the study period. Variables were analysed using Fisher's exact test or Wilcoxon rank-sum test as appropriate.

To assess the representativeness of our cohort we compared the socio-demographical characteristics of participants of our cohort to those retrieved from the 2010 French National Perinatal Survey [24]. The National Perinatal Surveys are conducted routinely in France and all births occurring during a given week in all maternity units in France are included. Women are interviewed between delivery and discharge about their sociodemographic characteristics, prenatal care and health behaviour. Data on health and obstetric care are collected from medical records. The sample is representative of all births at a national level [24].

To evaluate the participation to the study, two indicators: participation rate and percentage of "active participants" were calculated and compared to those of other (non-pregnant) participants to the general GrippeNet.fr cohort. For each participant, the participation rate was the number of WQ completed, divided by the length of follow-up (in weeks). The follow-up started at inclusion (registration on the platform) and ended at delivery. Participants were considered as "active participants" if their participation rate was above $15 \%$ (definition used in previous GrippeNet.fr and Influenzanet studies [12, 22, 25]). Indicators of participation were estimated in our sample and compared to those calculated among nonpregnant participants to the general cohort of GrippeNet.fr. Factors associated with active participation within the GGNET cohort were assessed using a multivariate logistic regression model. We first tested all sociodemographics and pregnancy-related variables in univariate analyses. Second, we entered explicative variables with a p-value $<0.20$ in a multivariate model. We then used a backward stepwise selection procedure (removal criteria: $p>0.05)$ to built the final model. Finally, the variable « participation to a previous season of GrippeNet.fr", which was a known factor of higher active participation in literature $[12,26]$, was added to the model as an adjustment variable. The results of both analyses are presented.

Statistical analysis was performed using $\mathrm{R}$ software (version 3.2) [27].

\section{Privacy and ethical approval}

Data were anonymous and participants were free to withdraw from the study at any time. This study was conducted in agreement with French regulations on privacy, data collection and treatment and was approved by the Comité consultatif sur le traitement de l'information en matière de recherche (CCTIRS, Advisory committee on information processing for research, authorisation 11.565) and by the Commission Nationale de l'Informatique et des Libertés (CNIL, French Data Protection Authority, authorisation DR-2012-024).

\section{Results \\ Participants}

Over the study period - November 19th 2014 and April 14th 2015-153 women were enrolled among 780000 eligible (coverage: 20 individuals per 100,000 pregnant women). Participation was widespread in the country, with participants coming from 53 out of 96 mainland French departments. Overall, mean age was 34 years (standard deviation, SD 4.3). One hundred thirty five (88 \%) had a university degree and $111(74 \%)$ were employed at the time of the study. Forty (27\%), 48 (32\%) and 61 (41\%) women were respectively in their first, second and third trimester of pregnancy. Nulliparity concerned 73 participants (48\%). Thirty-eight participants $(25 \%)$ gave birth during the influenza period. (Table 1)

\section{Representativeness}

Compared to the general population of French pregnant women, participants of the GGNET cohort were older (mean age 34.0 years (SD 4.3) vs. 29.7 years (SD 5.3)), more highly educated, more frequently employed and less frequently smokers (Table 1 ). On the other hand, no difference was found on Body Mass Index (BMI), parity or history of hospitalisation during a previous pregnancy.

\section{Participation}

During the study period, 152 pregnant women filled in at least one WQ, and a total of $1363 \mathrm{WQ}$ were completed. The mean time of follow-up was 9.0 weeks (SD 6.6). The median rate of participation was $78 \%$ (Inter Quartile Range IQR, 34-96) and 128 (84.\%) participants were considered as 'active participants'.

Compared to the other participants of GrippeNet.fr, the median rate of participation in pregnant participants to GGNET did not differ (78 \% (IQR, 34-96) vs. $87 \%$ (IQR, 45-96) respectively, p-value 0.37). Likewise, the percentage of active participants did not differ between the two cohorts ( $84 \%$ vs. $84 \%$, GGNET vs. GrippeNet.fr, respectively, p-value 0.92 ). (Table 2 )

Educational level and occupation were selected for the first step of the multivariate analysis of predictors of active participation. Participation to a previous GrippeNet.fr 
Table 1 Comparison of sample's characteristics of G-GrippeNet 2014/2015 with national statistics from the 2010 National Perinatal Survey

\begin{tabular}{|c|c|c|c|}
\hline & GGNET N (\%) & National perinatal survey 2010 N (\%) & $p$-value \\
\hline Total & 153 & 15187 & \\
\hline Age, years & & & $<10^{-3}$ \\
\hline$<25$ & $1(1 \%)$ & $2444(17 \%)$ & \\
\hline $25-29$ & $29(19 \%)$ & 4777 (33\%) & \\
\hline $30-34$ & $59(39 \%)$ & 4419 (31\%) & \\
\hline $35-39$ & $51(34 \%)$ & $2255(16 \%)$ & \\
\hline$>39$ & $12(8 \%)$ & $506(3 \%)$ & \\
\hline Educational Level & & & $<10^{-3}$ \\
\hline Middle School or less & $3(2 \%)$ & $3974(28 \%)$ & \\
\hline High School & $13(9 \%)$ & $2796(20 \%)$ & \\
\hline College or more & $135(89 \%)$ & $7290(52 \%)$ & \\
\hline Occupational status & & & $<10^{-3}$ \\
\hline Employed & $111(74 \%)$ & $9507(67 \%)$ & \\
\hline Housewife & $5(2 \%)$ & $1869(13 \%)$ & \\
\hline Student & $1(1 \%)$ & $344(2 \%)$ & \\
\hline Unemployed & $14(9 \%)$ & $1711(12 \%)$ & \\
\hline Other & $21(14 \%)$ & $718(5 \%)$ & \\
\hline Occupation (if employed) & & & $<10^{-3}$ \\
\hline Professional, manager, intermediate & $36(31 \%)$ & $4175(43 \%)$ & \\
\hline Office work & $64(55 \%)$ & $2714(29 \%)$ & \\
\hline Services & $5(4 \%)$ & $1713(18 \%)$ & \\
\hline Skilled manual work & $6(5 \%)$ & $453(5 \%)$ & \\
\hline Other manual work & 0 & $339(4 \%)$ & \\
\hline Other & $6(5 \%)$ & $88(1 \%)$ & \\
\hline Pregnancy trimester & & & - \\
\hline $1 s t$ & $40(27 \%)$ & - & \\
\hline 2nd & $47(32 \%)$ & - & \\
\hline $3 \mathrm{rd}$ & $61(41 \%)$ & - & \\
\hline Parity & & & 0.45 \\
\hline 0 & $73(48 \%)$ & $6396(43 \%)$ & \\
\hline 1 & $53(35 \%)$ & $5004(35 \%)$ & \\
\hline 2 & $20(13 \%)$ & $2069(14 \%)$ & \\
\hline$>2$ & $7(4 \%)$ & $1130(8 \%)$ & \\
\hline History of hospitalisation during previous pregnancy & & & - \\
\hline No & $82(78 \%)$ & - & \\
\hline Yes & $23(22 \%)$ & - & \\
\hline \multicolumn{4}{|l|}{ Chronic disease } \\
\hline No & $142(93 \%)$ & - & - \\
\hline Yes & $11(7 \%)$ & - & \\
\hline Asthma & 7 & - & \\
\hline Chronic respiratory disease & 2 & - & \\
\hline Chronic cardiac disease & 1 & - & \\
\hline
\end{tabular}


Table 1 Comparison of sample's characteristics of G-GrippeNet 2014/2015 with national statistics from the 2010 National Perinatal Survey (Continued)

\begin{tabular}{lll}
\hline Chronic renal disease & 1 & - \\
Diabetes & 0 & - \\
Immunosuppression & 1 & - \\
Body Mass Index before pregnancy & $11(7 \%)$ & \\
$<18.5$ & $106(70 \%)$ & $8811(65 \%)$ \\
$18.5-25$ & $26(17 \%)$ & $2360(17 \%)$ \\
$25-30$ & $8(6 \%)$ & $1347(10 \%)$ \\
$>30$ & & $11,679(83 \%)$ \\
Current smoker & $142(93 \%)$ & $2403(17 \%)$ \\
No & $11(7 \%)$ & 0.002 \\
Yes (1) &
\end{tabular}

(1) at enrollment in GGNET; during the 3rd trimester in the National Perinatal Survey

season was then included in the second step of the procedure. At the end of the model selection procedure, although not significant in the multivariate model, higher educational level and previous participation tended to be associated with an active participation (Odds Ratio, OR 2.52, IC95\% 0.70-8.11 and OR 2.93, IC95\% 0.90-13.27, respectively). (Table 3)

\section{Discussion}

GGNET is the first nationwide web-based influenza surveillance system specifically targeting pregnant women. For the first season in 2014/2015, 153 pregnant women were enrolled. Participants were distributed in more than half of French departments, differently from previous birth-cohorts approaches that were mainly local [18]. However, the sample was small (reaching a coverage of 20/100 000) and non-representative. Pregnant women of our cohort were older, with a higher degree of education and more frequently employed than the general population of pregnant women in France. Active participation was high and similar to that observed in participants of the general GrippeNet.fr cohort.

Only a few web-based studies have been conducted so far in the pregnant population. Most of them were large transversal studies assessing women behaviour or effects of interventions [16, 18, 28-30]. To our knowledge, no web-based survey has offered a longitudinal follow-up on a weekly basis. The strengths of our study are: a wide geographical distribution of participants at a national scale, and a high active participation rate. The latter being probably related to the simplicity of follow-up (taking only few minutes per week with weekly automatic email reminders).

Main limitations are the small sample size and the non-representativeness of the sample. Despite our efforts, we calculated that our sample represented only $0.02 \%$ of French women who were pregnant during the study period. Our communication campaign was based on both online (web sites dedicated to pregnant women and young mothers' health) and offline methods (advertising posters displayed in waiting rooms of large maternity centres). Among women who agreed to participate, half reported to have heard about the study via the Internet and only $15 \%$ through health care workers. This suggests that the Web and associated online media (e.g. mobile phone app) are of crucial importance to assemble a large cohort and ensure high participation for the next influenza seasons. Moreover, previous work has shown that in France, contrary to what observed in other Influenzanet countries, participants recruited through online communication have a higher follow-up participation [31]. The lack of representativeness of our sample, likely induced by the non-representative nature of the Internet population, thus translating into coverage biases, and the self-selection of participants, also called the 'volunteer effect' (i.e. those who choose to volunteer for studies

Table 2 Comparison of participation between pregnant participants of G-GrippeNet and general participants of GrippeNet.fr 2014/2015

\begin{tabular}{llll}
\hline & GGNET & General Participants & $p$-value \\
\hline Number of participants & 153 & 6574 & 89172 \\
Number of weekly questionnaire & 1363 & 13 & \\
Mean number of weekly questionnaires & 9 & $87 \%(45-96 \%)$ & 0.37 \\
Median rate of participation (IQR) & $78 \%(34-96 \%)$ & $5504(84 \%)$ & 0.92 \\
Active participants (\%) & $128(84 \%)$ & \\
\hline
\end{tabular}


Table 3 Rate of active participant in the 2014/2015 G-GrippeNet cohort depending on characteristics, and factors associated with active participation (= participation rate $>15 \%$ ) in univariate and multivariate logistic regression

\begin{tabular}{|c|c|c|c|c|c|c|c|}
\hline & $N(\%)$ & OR $\left[95 \% \mathrm{Cl}^{\mathrm{a}}\right.$ & $p$-value & ORa $[95 \% \mathrm{Cl}]^{* *}$ & $p$-value & ORa $[95 \% \mathrm{Cl}]^{* * *}$ & $p$-value \\
\hline Total of active participants & 128/153 (84 \%) & - & - & - & - & - & - \\
\hline \multicolumn{8}{|l|}{ Age, years } \\
\hline$<35$ & $60 / 74(82 \%)$ & 1 & 0.32 & - & - & - & - \\
\hline $35+$ & 67/77 (87 \%) & $1.56[0.65-3.88]$ & & - & & - & \\
\hline \multicolumn{8}{|l|}{ Educational Level } \\
\hline Less than High school & 11/16 (69 \%) & 1 & 0.09 & 1 & 0.15 & 1 & 0.13 \\
\hline High school or more & 116/135 (86 \%) & $2.77[0.80-8.59]$ & & $2.41[0.68-7.68]$ & & $2.52[0.70-8.11]$ & \\
\hline \multicolumn{8}{|l|}{ Occupation } \\
\hline Professional & 28/32 (88 \%) & 1 & 0.09 & 1 & 0.13 & 1 & 0.19 \\
\hline Office work & $56 / 62(90 \%)$ & $1.33[0.32-5.05]$ & & $1.41[0.34-1.61]$ & & $1.21[0.28-4.75]$ & \\
\hline Other & 44/58 (76 \%) & $0.45[0.12-1.40]$ & & $0.51[0.13-1.61]$ & & $0.53[0.14-1.69]$ & \\
\hline \multicolumn{8}{|l|}{ Pregnancy trimester } \\
\hline 1 st \& 2nd & 72/87 (83 \%) & 1 & 0.68 & - & - & - & - \\
\hline $3 r d$ & $52 / 61(85 \%)$ & $1.20[0.50-3.06]$ & & - & & - & \\
\hline \multicolumn{8}{|l|}{ Parity } \\
\hline 0 & 63/73 (86 \%) & 1 & 0.49 & - & - & - & - \\
\hline$\geq 1$ & 65/79 (82 \%) & $0.74[0.30-1.77]$ & & - & & - & \\
\hline \multicolumn{8}{|l|}{ Chronic disease } \\
\hline No & $120 / 142(85 \%)$ & 1 & 0.71 & - & - & - & - \\
\hline Yes & 8/10 (80 \%) & $0.73[0.17-5.10]$ & & - & & - & \\
\hline \multicolumn{8}{|c|}{ Previous participation to GrippeNet.fr } \\
\hline No & 86/107 (80 \%) & 1 & 0.03 & - & - & 1 & 0.10 \\
\hline Yes & $42 / 45(93 \%)$ & $3.41[1.12-15.05]$ & & - & & $2.93[0.90-13.27]$ & \\
\hline
\end{tabular}

may differ in lifestyle and health from those who decline) [11] was similarly observed for the entire Grippenet.fr cohort in comparison to the general population. In previous GrippeNet.fr seasons, other authors reported a large underrepresentation of the youngest and oldest age classes in the participants compared to the French population [12, 25]. These findings were also reported in web-based birth cohorts [18]. This underlines that the next communication campaigns should preferentially target younger pregnant women. Again, the use of new, Internet-based communication channels is likely to have the largest impact in this population.

In this study, the active participation rate was high and factors that we found associated with active participation in multivariate analysis are in line with previous research that has shown that different factors can influence participation rate. Socio-demographic factors, such as low educational level or unemployment, as well as unhealthy life style factors (smoking, alcohol consumption) are typically related to increased risk of non-response and attrition in epidemiological studies [32, 33]. This was also observed in a large study on the European Influenzanet cohort [26]. Longitudinal studies are important in public health research for identifying risk factors related to negative health outcomes. However, a major concern in such studies is that the longer the follow-up period, the higher are the chances for dropout. In our study, active participation of pregnant women was similar to that observed in participants of the general GrippeNet.fr cohort.

Social medias and widening of the Internet coverage have opened the way to real-time collaborative data collection and led to the development of innovative approaches for disease surveillance. This longitudinal web-based surveillance study proved feasible in pregnant women and will bring original data on the epidemiology of influenza in this population. It will be helpful to promote vaccination in this population.

\section{Conclusions}

We report the results of the first prospective cohort study aiming to describe influenza epidemic in pregnant women. Despite the small sample size and lack of representativeness 
on certain demographic variables, the active participation rate was high, suggesting that pregnant women are prone to adhere to a longitudinal follow-up of their health status via the Internet. In multivariate analysis, higher educational level and previous participation tended to be associated with an active participation. To reach younger participants with mixed educational level, it also seems crucial, besides traditional channels, to encourage enrolment via online social media sites and apps.

\section{Competing interest}

The authors declare that they have no competing interest.

\section{Authors' contribution}

All authors contributed to the design and implementation of the study; PL, CG, CT and SK analysed the data, PL and SK wrote the first draft; and all authors reviewed and edited the manuscript. All authors read and approved the final manuscript.

\section{Acknowledgment}

We are very grateful to the following people and institutions who made significant contributions to the running of the study: Armelle Telang from the National Network of Site Management Organization in Obstetrics and Gynecology (GO-CIC), Nezha Lenzi and Mohammed Rahoui from the Innovative Clinical Research Network in Vaccinology (I-REIVAC), Véronique Tessier from the DHU Risks in Pregnancy and Albane Tresse from the Premup foundation.

\section{Funding}

This work was supported by the French National Research Agency (HARMSFLU, grant number ANR-12-MONU-0018), a grant from the Société de Pathologie Infectieuse de Langue Française (SPILF) and a postgraduate studentship award from the Agence Regionale de Santé Île-de-France.

\section{Author details \\ ${ }^{1}$ Sorbonne Universités, UPMC Univ Paris 06, INSERM, Institut Pierre Louis d'épidémiologie et de Santé Publique (IPLESP UMRS 1136), F75012 Paris, France. ${ }^{2}$ Department of Infectious diseases, CIC Cochin-Pasteur, Cochin Broca Hôtel-Dieu hospital, Assistance Publique-Hôpitaux de Paris (AP-HP), Paris, France. ${ }^{3}$ INSERM U 1153, Obstetrical, Perinatal and Pediatric Epidemiology Research Team (EPOPé), Center for Epidemiology and Statistics Sorbonne Paris Cité, DHU Risks in pregnancy, Paris Descartes University, Paris, France. ${ }^{4}$ INSERM CIC 1417, Paris, France. ${ }^{5}$ Inserm, F-CRIN, Innovative clinical research network in vaccinology (I-REIVAC), Paris, France. ${ }^{6} \mathrm{Clinical}$ Investigation Center (CIC) INSERM 1432, Gynecology and Obstetrics CIC Network (GO-CIC) \& Hepato-gastroenterology department, Dijon Hospital, Dijon, France. ${ }^{7}$ Department of Infectious Diseases, Institut de Veille Sanitaire (InVS), St Maurice, France. ${ }^{8}$ Department of Obstetrics and Gynecology, Cochin Broca Hôtel-Dieu hospital, Assistance Publique-Hôpitaux de Paris (AP-HP), Paris, France.}

\section{Received: 26 October 2015 Accepted: 19 February 2016}

\section{Published online: 11 March 2016}

\section{References}

1. Dodds L, McNeil SA, Fell DB, Allen VM, Coombs A, Scott J, et al. Impact of influenza exposure on rates of hospital admissions and physician visits because of respiratory illness among pregnant women. CMAJ. 2007;176:463-8.

2. Irving WL, James DK, Stephenson T, Laing P, Jameson C, Oxford JS, et al. Influenza virus infection in the second and third trimesters of pregnancy: a clinical and seroepidemiological study. BJOG Int J Obstet Gynaecol. 2000;107:1282-9.

3. Neuzil KM, Reed GW, Mitchel EF, Simonsen L, Griffin MR. Impact of influenza on acute cardiopulmonary hospitalizations in pregnant women. Am J Epidemiol. 1998;148:1094-102.

4. HARRIS JW. Influenza occurring in pregnant women: a statistical study of thirteen hundred and fifty cases. J Am Med Assoc. 1919;72:978-80.

5. Griffith GW, Adelstein AM, Lambert PM, Weatherall JA. C. Influenza and Infant Mortality. BMJ. 1972;3:553-6.
6. Pierce M, Kurinczuk JJ, Spark P, Brocklehurst $P$, Knight M, UKOSS. Perinatal outcomes after maternal 2009/H1N1 infection: national cohort study. BMJ. 2011;342:d3214

7. WHO | World Health Organization [Internet]. WHO [cité 2015 mai 5]; Available from: http://www.who.int/influenza/surveillance_monitoring/en/. Accessed 5 May 2015

8. Valleron AJ, Bouvet E, Garnerin P, Ménarès J, Heard I, Letrait S, et al. A computer network for the surveillance of communicable diseases: the French experiment. Am J Public Health. 1986;76:1289-92.

9. French GPs Sentinelles network > France > The Sentinelles network [Internet]. [cité 2015 août 12]; Available from: https://websenti.u707.jussieu. $\mathrm{fr} /$ sentiweb/?page=presentation. Accessed 12 Aug 2015.

10. van Gelder MMHJ, Bretveld RW, Roeleveld N. Web-based questionnaires: the future in epidemiology? Am J Epidemiol. 2010;172:1292-8.

11. Eysenbach G. Infodemiology and infoveillance: framework for an emerging Set of public health informatics methods to analyze search, communication and publication behavior on the internet. J Med Internet Res. 2009;11, e11.

12. Debin M, Turbelin C, Blanchon T, Bonmarin I, Falchi A, Hanslik T, et al. Evaluating the feasibility and Participants' representativeness of an online nationwide surveillance system for influenza in France. PLoS ONE. 2013;8:e73675.

13. Debin M, Colizza V, Blanchon T, Hanslik T, Turbelin C, Falchi A Effectiveness of 2012-2013 influenza vaccine against influenza-like illness in general population: Estimation in a French web-based cohort. Hum. Vaccines Immunother. [Internet] 2014 [cité 2014 mai 8];10. Available from: http://www.landesbioscience.com/journals/vaccines/article/27439/. Accessed 8 May 2015.

14. Pandolfi E, Agricola E, Gonfiantini MV, Gesualdo F, Romano M, Carloni E, et al. Women participating in a web-based preconception study have a high prevalence of risk factors for adverse pregnancy outcomes. BMC Pregnancy Childbirth. 2014;14:169.

15. Allais G, Rolando S, De Lorenzo C, Manzoni GC, Messina P, Benedetto C, et al. Migraine and pregnancy: an internet survey. Neurol Sci Off J Ital Neurol Soc Ital Soc Clin Neurophysiol. 2013;34 Suppl 1:S93-9.

16. Evans WD, Wallace Bihm J, Szekely D, Nielsen P, Murray E, Abroms L, et al. Initial Outcomes From a 4-Week Follow-Up Study of the Text4baby Program in the Military Women's Population: Randomized Controlled Trial. J. Med. Internet Res. [Internet] 2014 [cité 2015 mai 15];16. Available from: http://www.ncbi.nlm.nih.gov/pmc/articles/PMC4051747/. Accessed 15 May 2015.

17. Firestone R, Cheng S, Pearce N, Douwes J, Merletti F, Pizzi C, et al. Internetbased birth-cohort studies: is this the future for epidemiology? JMIR Res Protoc. 2015:4, e71.

18. Richiardi L, Baussano I, Vizzini L, Douwes J, Pearce N, Merletti F. Feasibility of recruiting a birth cohort through the Internet: the experience of the NINFEA cohort. Eur J Epidemiol. 2007;22:831-7.

19. Ding H, Black CL, Ball S, Donahue S, Izrael D, Williams WW, et al. Influenza vaccination coverage among pregnant women-United States, 2013-14 influenza season. MMWR Morb Mortal Wkly Rep. 2014;63:816-21.

20. Centers for Disease Control and Prevention (CDC). Influenza vaccination coverage among pregnant women-United States, 2012-13 influenza season. MMWR Morb Mortal Wkly Rep. 2013;62:787-92

21. Influenzanet: Home [Internet]. [cité 2015 mai 5]; Available from: https:// www.influenzanet.eu/. Accessed 5 May 2015.

22. Paolotti D, Carnahan A, Colizza V, Eames K, Edmunds J, Gomes G, et al. Web-based participatory surveillance of infectious diseases: the Influenzanet participatory surveillance experience. Clin Microbiol Infect Off Publ Eur Soc Clin Microbiol Infect Dis. 2014;20:17-21.

23. Cantarelli P, Debin M, Turbelin C, Poletto C, Blanchon T, Falchi A, et al. The representativeness of a European multi-center network for influenza-likeillness participatory surveillance. BMC Public Health. 2014;14:984.

24. Insee - Population - En 2013, 811510 bébés sont nés en France - Plus de 800000 bébés par an depuis 2005 [Internet]. [cité 2015 août 31]; Available from: http://www.insee.fr/fr/themes/document.asp?ref_id=if9. Accessed 31 Aug 2015.

25. Blondel B, Lelong N, Kermarrec M, Goffinet F. National Coordination Group of the National Perinatal Surveys. Trends in perinatal health in France from 1995 to 2010. Results from the French National Perinatal Surveys. J Gynécologie Obstétrique Biol Reprod. 2012;41:e1-15.

26. Bajardi P, Vespignani A, Funk S, Eames KT, Edmunds WJ, Turbelin C, et al. Determinants of follow-Up participation in the internet-based European influenza surveillance platform influenzanet. J Med Internet Res. 2014;16, e78. 
27. R: The R Project for Statistical Computing [Internet]. [cité 2015 oct 5]; Available from: https://www.r-project.org/. Accessed 5 Oct 2015.

28. Lupattelli A, Spigset O, Twigg MJ, Zagorodnikova K, Mårdby AC, Moretti ME, et al. Medication use in pregnancy: a cross-sectional, multinational web-based study. BMJ Open. 2014;4, e004365.

29. Adolfsson A, Linden K, Sparud-Lundin C, Larsson P-G, Berg M. A web-based support for pregnant women and new mothers with type 1 diabetes mellitus in Sweden (MODIAB-Web): study protocol for a randomized controlled trial. Trials. 2014;15:513.

30. Herbec A, Brown J, Tombor I, Michie S, West R. Pilot randomized controlled trial of an internet-based smoking cessation intervention for pregnant smokers ('MumsQuit'). Drug Alcohol Depend. 2014;140:130-6.

31. Bajardi P, Paolotti D, Vespignani A, Eames K, Funk S, Edmunds WJ, et al. Association between recruitment methods and attrition in internet-based studies. PLoS ONE. 2014;9, e114925.

32. Nilsen RM, Vollset SE, Gjessing HK, Skjærven R, Melve KK, Schreuder P, et al. Self-selection and bias in a large prospective pregnancy cohort in Norway. Paediatr Perinat Epidemiol. 2009;23:597-608.

33. Van Loon AJM, Tijhuis M, Picavet HSJ, Surtees PG, Ormel J. Survey nonresponse in the Netherlands: effects on prevalence estimates and associations. Ann Epidemiol. 2003;13:105-10.

\section{Submit your next manuscript to BioMed Central} and we will help you at every step:

- We accept pre-submission inquiries

- Our selector tool helps you to find the most relevant journal

- We provide round the clock customer support

- Convenient online submission

- Thorough peer review

- Inclusion in PubMed and all major indexing services

- Maximum visibility for your research

Submit your manuscript at www.biomedcentral.com/submit 